\title{
The Review
}

\section{Inventing the wheel in general practice}

As multiple morbidity becomes more common, budgets tighten and inequalities widen, the fragmentation of health care is increasingly prevalent, expensive, and unacceptable.

Fragmentation of care occurs when encounters with patients address only some of a patient's problems, when practitioners are not informed by previous encounters and do not inform the encounters which follow, when the left hand of the service does not know what the right hand is doing and when care is delivered on a partial basis, for example, by responding primarily to demands rather than to needs.

Fragmentation of health systems often occurs in conurbations, when some services are delivered on the basis of GP lists, while other services are organised at an area level. Federations of general practices can fragment care within areas when only some practices are involved.

A consistent conclusion of Deep End meetings has been that general practices offer a ready solution to the problems of fragmentation, as the natural and sustainable hubs around which local health systems should develop. Although general practices are not the only part of the NHS providing contact, coverage, and continuity, no other part of the NHS offers these features for so many people, in a manner that consistently gains public approval and which has stood the test of time.

Consultations are the heart of general practice and cumulatively provide not only high levels of population coverage, but also continuity of contact with individuals, allowing the development of relationships and trust. They also provide serial starting points for anticipatory care, after current problems have been addressed.

However, while consultations are essential, they are no longer sufficient. GPs cannot do everything and have no wish to do so. Consultations provide opportunities to instigate referral to other professionals and services, either inside the practice, within the local community or further afield.

Pursuing the wheel analogy, hubs need connections to rims, spokes and axles. GPs must become wheelwrights. The inner wheel of general practice links practitioner consultations with the activities of other members of the team. When several team members accumulate important information about patients and their problems, the 'spokes' of the wheel are regular channels of communication within the team. The outer wheel of general practice links practitioner consultations with many other NHS and local authority services within the local community. Some practices also link to non-medical community resources for the help they can provide for patients ('social prescribing').'

In deprived areas, the spokes connecting hubs and other local services have to be short. A consistent finding from many meetings of Deep End practitioners is that if referral is not timely, local, and to a familiar person or setting, patients are less likely to attend. Therefore, attached workers are in demand, for example working in mental health, alcohol and addiction, or child surveillance.

Attached workers are not a panacea, and as with all aspects of joint working, their usefulness depends on productive professional relationships, based on personal contact, positive experiences, regular communication, reciprocity, and trust. Deep End practitioners report good and bad examples of attached workers in practice.

The challenge is not only how general practice hubs relate to the rest of the local health system, but also how area-based services relate to all of the practices within their area. For example, in the Deep End meeting on Alcohol Problems in Young Adults, GPs were impressed by the range and quality of services provided by area based community addiction teams. However, by their own admission, such teams deal with only about $40 \%$ of people with severe alcohol problems in their areas. ${ }^{2}$ Health systems audit needs to take account of $100 \%$ of the population needing care.

Every local system depends on the sum of the relationships of which it is comprised, including relationships between practices and other professionals and services. Currently, there is little mapping or review of these relationships, although their quality is often well known to everyone at ground level and frequently determines how services are provided and used. Gang culture is not only a feature of deprived housing estates. It features widely in public services.

When such relationships are reviewed, a hierarchy emerges, ranging from no knowledge, to knowledge but no contact, to cooperation on a scale from high to low

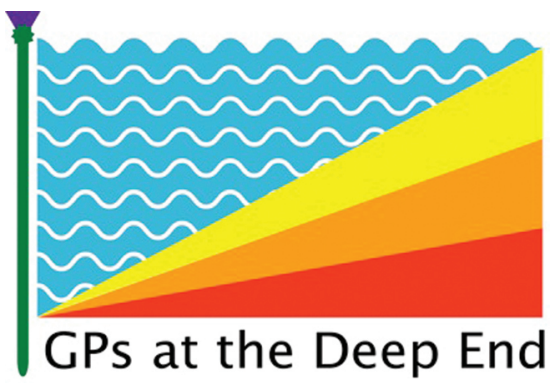

volume and a spectrum from good to bad results. Genuine collaboration occurs when joint working is valued at every level, and planned and reviewed on a regular basis. Neither general practices nor area-based services can do this on their own. It takes two to tango

An intrinsic feature of general practice, in contrast to specific care programmes, is that hub, spoke, and rim relationships are needed for many different groups of patients, including those with cardiovascular disease risks, multiple morbidity, problems of old age, and vulnerable families. With a full compliment of wheels, connected, balanced, and serviced, general practice need not be stuck in the present. It could transport health care into the future.

\section{Graham Watt,}

On behalf of the Deep End Steering Group. This is the 11 th in a series of articles from General Practitioners at the Deep End.

DOI: 10.3399/bjgp11X606708

\section{ADDRESS FOR CORRESPONDENCE}

Graham Watt

General Practice and Primary Care, College of Medical, Veterinary and Life Sciences University of Glasgow, 1 Horselethill Road, Glasgow, G12 9LX, UK.

\section{E-mail: Graham.Watt_aglasgow.ac.uk}

\section{REFERENCES}

1. Deep End Report 9. Learning Journeys. http://www.gla.ac.uk/media/media 179092 e n.pdf (accessed 6 Oct 2011).

2. Deep End Report 11. Alcohol problems in adults under 40 http://www.gla.ac.uk/media/media_179503_e n.pdf laccessed 6 Oct 2011). 